\title{
MINIATURIZED TEXTILE-BASED MULTI-LAYER PH-SENSOR FOR WOUND MONITORING APPLICATIONS
}

\author{
Andreas Nocke', Anna Schröter², Chokri Cherif', Gerald Gerlach² \\ ${ }^{1}$ Institute of Textile Machinery and High Performance Material Technology (ITM), Technische Universität Dresden, Dresden, \\ Germany, e-mail: andreas.nocke@tu-dresden.de, chokri.cherif@tu-dresden.de \\ ${ }^{2}$ Solid-State Electronics Laboratory (IFE), Technische Universität Dresden, Dresden, Germany, \\ e-mail: anna.schroeter@tu-dresden.de, gerald.gerlach@tu-dresden.de
}

Abstract:

Wound assessment has become an important issue in the wound treatment procedure. One important indicator of the wound status is the $\mathrm{pH}$ value. Our approach to assess this quantity is through use of a fiber sensor coated with a $\mathrm{pH}$-responsive hydrogel, which functions as a sensitive layer for impedance measurements. An advantage of this is its integratability into wound dressings using standard textile technologies. The $\mathrm{pH}$ characteristic shows a $\mathrm{pH}$-dependent behavior of the absolute impedance at certain frequencies. The fabrication technology and sensor characteristics are discussed. The values of almost $14 \%$ impedance change demonstrate the potential for improvement by optimizing fabrication technologies. The presented sensor meets all requirements necessary for wound $\mathrm{pH}$ assessment.

\section{Key words:}

Wound monitoring, $\mathrm{pH}$ sensor, textile technology, multi-layer fiber .

\section{Introduction}

To reduce the possibility of complications in the wound healing process, a continuous sensor-based monitoring of the healing area is necessary. With this acquired sensory information, possible complications typically resulting from infections of the wound, can be treated promptly so that penetration of pathogens into the body and the bloodstream can be avoided. Inflammation is a complex cellular process that changes the properties of the wound exudate and alters various physiological parameters like; $\mathrm{pH}$ [1], temperature [2-3], wound humidity or viscosity [4-5], concentration of various ions (particularly reactive oxygen species) [6-7] and content of lactate [8-9] or urea [10-11]. Therefore, the wound healing process can be assessed by continuous monitoring of one or more properties.

A potentiometric wound sensor for the measurement of uric acid concentrations based on carbon fibers has been described by Sharp and Davis [10-11]. The principle is based on the oxidation of uric acid in the carbon fibers, detected by applying a voltage sweep. This method operates in the low frequency range with comparatively high voltages $(230 \mathrm{mV})$, and up to now, the stability and reproducibility of the sensor has been inadequate.

Another approach is the integration of optical fibers surrounded by functional hydrogels, which exhibit a change in their surface properties or turgidity, in response to reactive proteins and $\mathrm{pH}$ [12]. This leads to a change in the refractive index, and thus to a change of the evanescent field of the light guide. The optical analysis demands high standards for processing accuracy of the optical set-up, thus making physiological application complicated and costly.

Conolly et al., developed a system for monitoring moisture in wound dressings with integrated textile sensors using a printing technology [4-5]. The moisture level evaluation is carried out by impedance measurement, but the large-scale arrangement of the electrodes might lead to measurements of spurious artifacts, such as air pockets.
In this paper a novel, miniaturized, textile-based and biocompatible sensor set-up for the detection of $\mathrm{pH}$-changes is presented. The $\mathrm{pH}$ value plays an important role in the wound healing process and the precise relationship between the $\mathrm{pH}$ value and the condition of the wound depends on the wound type. Generally, it can be said that a permanent $\mathrm{pH}$-value higher than 7.5 over a long period, is an indicator of impaired wound healing [1].

\section{Methods and Materials}

The presented textile-based $\mathrm{pH}$-sensor is designed to fulfill the following demands:

(i) integratability into the dressing using standard textile technologies,

(ii) high degree of flexibility appropriate for the application,

(iii) simple electrical readout of the sensor characteristics by using a direct electrical measurement system,

(iv) good long-term stability.

A high stability of the sensor setup is achieved by the integration of the electrodes required for the impedance measurement in a one-thread construction (Figure 1). Therefore, lateral displacement of the electrodes, relative to each other, does not occur. The required electrical insulation of the electrodes and $\mathrm{pH}$-selectivity of the sensor is achieved by using a $\mathrm{pH}$ sensitive hydrogel layer between the electrodes.

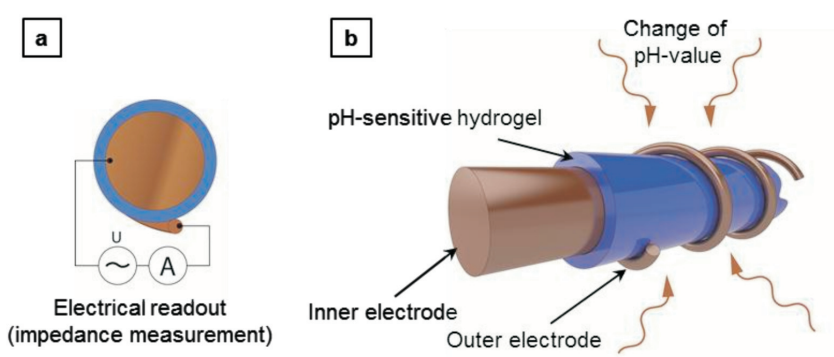

Figure 1. Schematic view of the impedimetric textile-based multi-layer fiber sensor for wound $\mathrm{pH}$-detection: a) cross-section, and b) perspective view. 
The materials used were selected to; (i) ensure the required electrical and chemical properties, and (ii) be biocompatible. Gold wires with a diameter of $50 \mu \mathrm{m}$ (supplied by Heraeus) are used as the material of the inner and outer electrode. The electrical insulation and $\mathrm{pH}$-sensitive layer consists of the hydrogel poly(vinyl alcohol) / poly(acrylic acid) (PVA/PAA) with a $\mathrm{pH}$-dependent swelling behavior in the range of $\mathrm{pH} 5$ to $\mathrm{pH} 11$ [13]. PVA and PAA were purchased from Sigma Aldrich and used as received. The preparation of the pregel solution was performed as described in [14].

The process of fabricating the $\mathrm{pH}$-sensitive multi-layer fiber sensor involved the following steps:

- fixation of a gold wire (inner electrode) in a tension frame (Figure 2),

- dip coating the gold wire in a pregel solution (7.5 wt.\% PVA/ PAS in $\mathrm{H}_{2} \mathrm{O}$ ) and subsequent thermal crosslinking of the PVA/ PAA in an oven $\left(130^{\circ} \mathrm{C}, 30 \mathrm{~min}\right)$,

- manual enwinding of a second gold wire (outer electrode) and

- coating and thermal crosslinking of an additional PVA/PAAlayer for mechanical stabilization and immobilization of the gold wires.

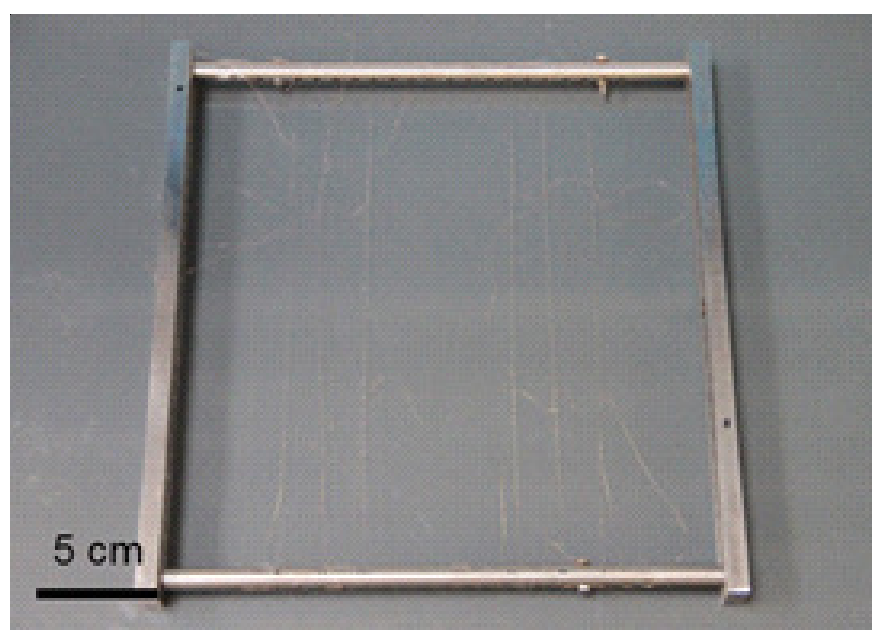

Figure 2. Tension frame with $\mathrm{pH}$-sensitive multi-layer fiber sensor layer after production process.

After the sensors have been separated into single sensor elements, the inner and outer wires were connected to an impedance analyzer (Sciospec ISX-3). Measurements of the impedance were performed in a frequency range from $1 \mathrm{~Hz}$ to $1 \mathrm{MHz}$. The sensor was calibrated at $\mathrm{pH} 6$. To adjust the $\mathrm{pH}$ values, $1 \mathrm{M}$ phosphor buffered saline (PBS) solution was mixed with hydrogen chloride or sodium peroxide (all purchased from Sigma Aldrich). The $\mathrm{pH}$ value was immediately checked with a pH meter (Calimatic 765) following every impedance measurement.

\section{Results and Discussion}

Figure 3 depicts the setup of a pH-sensitive multi-layer fiber sensor and a typical impedance spectrum measured with this hydrogel wire sensor is shown in Figure 4(a). The behavior of the sensor is capacitive with ohmic losses. At lower frequencies the sensor has a resistance of $172 \mathrm{k} \Omega$, which is close to the DC resistance. This value is mostly related to electrical pathways between the electrodes which consist of the hydrogel and the buffer liquid containing ions. In nearly DC conditions, these ions create a Helmholtz double layer, which is highly

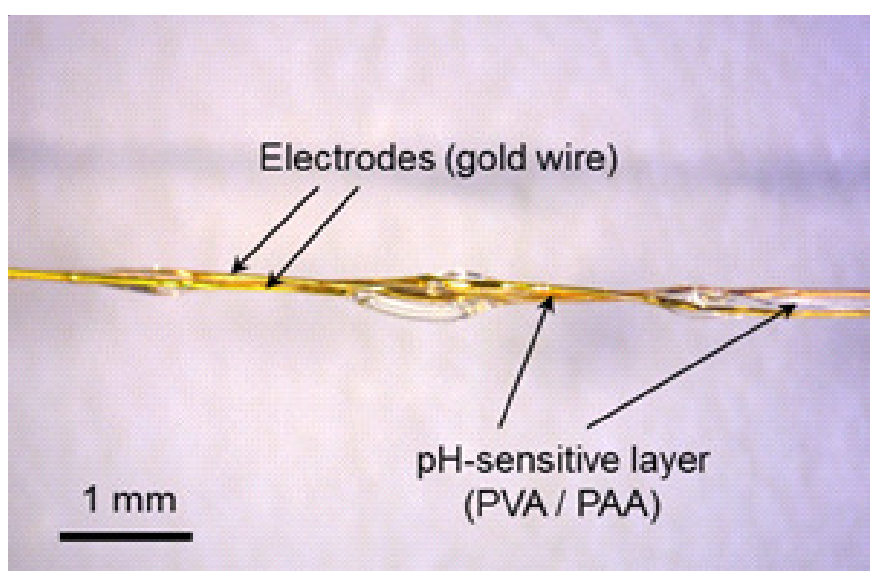

Figure 3. Microscopic image of the impedimetric multi-layer fiber sensor with $\mathrm{pH}$-sensitive functional layer.

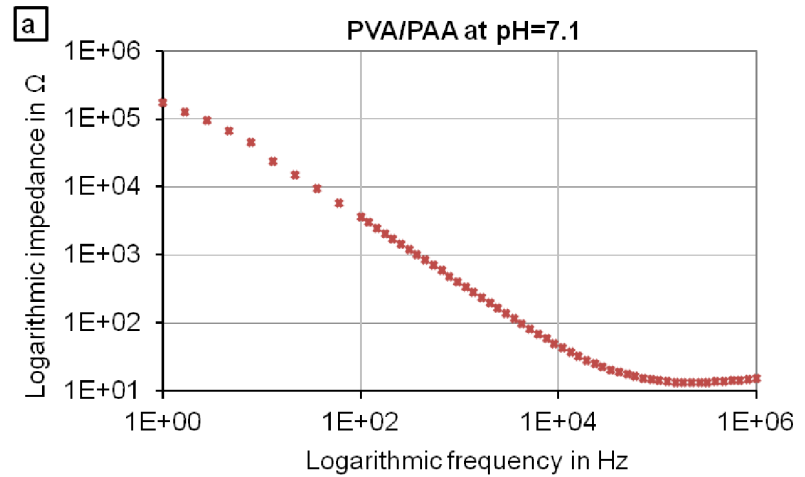

b

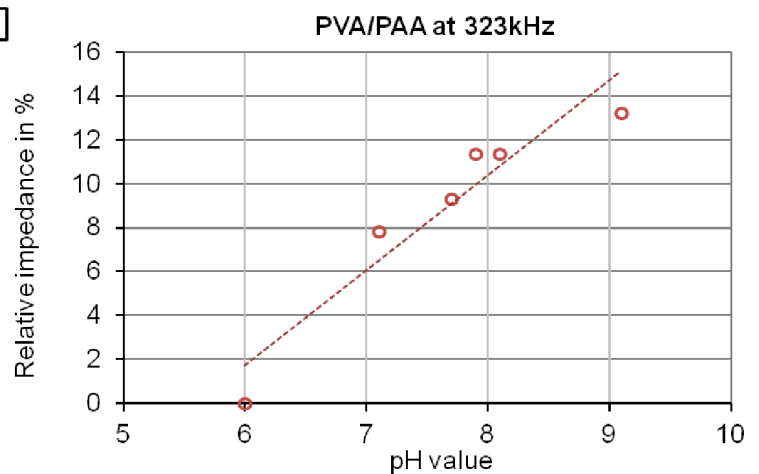

Figure 4. a) Impedance spectra of the $\mathrm{pH}$-sensitive fiber sensor at a $\mathrm{pH}$ value of 7.1 , and $\mathrm{b}$ ) relative impedance change of multi-layer fiber sensor depending on $\mathrm{pH}$ value at $323 \mathrm{kHz}$ excitation frequency.

isolating. The resistance of the sensor is then determined by the hydrogel structure. The capacitive behavior afterwards is caused by the ionic movements in the liquid and leads to a drop of the curve. At about $100 \mathrm{kHz}$, the impedance is constant because ionic movements cannot follow the field changes. In this region, the impedance depends only on the water-dipole elongation. Over the whole spectrum, impedance is strongly related to the distance between the electrodes and this distance changes due to the $\mathrm{pH}$ dependent swelling behavior of the hydrogel.

Impedance spectra indicate that a $\mathrm{pH}$ sensitivity occurs at a frequency of about $323 \mathrm{kHz}$. Figure 4(b) displays the relative impedance change of the absolute impedance of PVA/PAA over $\mathrm{pH}$ at this frequency. From $\mathrm{pH} 6$ to $\mathrm{pH} 9.1$ the absolute impedance increases by almost $14 \%$. The physical modeling of this effect is complicated due to an undefined geometric configuration and associated field distributions. Also, the 
hydrogel swelling is not defined precisely in this case and could lead to a mechanical destruction of the hydrogel matrix by the gold wires under extreme conditions. These effects have to be considered for further investigations.

\section{Conclusions}

We assessed the ability of a $\mathrm{pH}$-sensitive multi-layer fiber sensor for wound monitoring. This quite simple approach achieved very promising results for a $\mathrm{pH}$ response of the sensor in a physiological relevant range. A defined geometry and increasing number of windings leads to a higher sensitivity and reproducibility. This can be achieved through automatic fabrication of the windings, e.g., with a twine machine. Implementing an adhesion promoter and decreasing the hydrogel thickness to a minimum should considerably increase the output signal. Next steps will include the optimization of adhesion, hydrogel deposition and electrode placements. This will be accompanied by characterization and modeling of the sensor behavior. Finally the sensor has to be tested in in vivo experiments on animal wound models.

\section{References:}

1. Schneider, L.; Korber, A.; Grabbe, S.; Dissemond, J.: Influence of $\mathrm{pH}$ on wound-healing: a new perspective for wound-therapy? Archives of Dermatological Research 298(2007)9, pp. 413-420.

2. Armstrong, D. G.; Lavery, L. A.; Liswood, O. J.; Todd, W. F.; Tredwell, J. A.: Infrared Dermal Thermometry for the HighRisk Diabetic Foot. Physical Therapy 77(1997)2, pp. 169175.

3. Bharara, M.; Schoess, J.; Nouvong, A.; Armstrong, D. G. Wound inflammatory index: a "proof of concept" study to assess wound healing trajectory. Journal of Diabetes Science and Technology 4(2010), pp. 773-779.

4. Connolly, P.; McColl, D. (18.04.2005): Wound Dressings and Performance Measurement of Such Dressings. US020080171957A1.

5. McColl, D.; Cartlidge, B.; Connolly, P.: Real-time monitoring of moisture levels in wound dressings in vitro: An experimental study. International Journal of Surgery 5(2007)5, pp. 316-322.

6. Test, S. T.; Weiss, S. J.: Quantitative and temporal characterization of the extracellular $\mathrm{H} 2 \mathrm{O} 2 \mathrm{pool}$ generated by human neutrophils. Journal of Biological Chemistry 259(1984)1, pp. 399-405

7. [7] Liu, X.; Zweier, J. L.: A real-time electrochemical technique for measurement of cellular hydrogen peroxide generation and consumption: evaluation in human polymorphonuclear leukocytes. Free Radical Biology and Medicine 31(2001)7, pp. 894-9018.

8. Cho, M.; Hunt, T. K.; Hussain, M. Z.: Hydrogen peroxide stimulates macrophage vascular endothelial growth factor release. American Journal of Physiology - Heart and Circulatory Physiology 280(2001)5, pp. H2357-H2363.

9. Trabold, O.; Wagner, S.; Wicke, C.; Scheuenstuhl, H.; Hussain, M. Z.; Rosen, N.; Seremetiev, A.; Becker, H. D.; Hunt, T. K.: Lactate and oxygen constitute a fundamental regulatory mechanism in wound healing. Wound Repair and Regeneration 11(2003)6, pp. 504-509.

10. Sharp, D.; Davis, J.: Integrated urate sensors for detecting wound infection. Electrochemistry Communications 10(2008) 5, pp. 709-713.
11. Sharp, D.; Forsythe, S.; Davis, J.: Carbon fibre composites: integrated electrochemical sensors for wound management. Journal of Biochemistry 144(2008)1, pp. 8793.

12. Vincenzini, P.; Rossi, D. de: Wearable biosensors for monitoring wound healing. Advances in Science and Technology 57(2008), pp. 80-87.

13. Schulz, V.; Gerlach, G; Günther, M.; Magda, J. J.; Solzbacher, F.: Piezoresistive $\mathrm{pH}$ microsensors based on stimulisensitive polyelectrolyte hydrogels. Technisches Messen 77(2010)3, pp. 179-186.

14. Richter, A.; Bund, A.; Keller, M.; Arndt, K. - F.: Characterization of a microgravimetric sensor based on $\mathrm{pH}$ sensitive hydrogels. Sensors and Actuators B: Chemical 99(2004)23, pp. 579-585. 